\title{
Algebre senza base finita (II) $\left(^{1}\right)$.
}

\author{
Memoria di Iacopo BarsotTi (a l'isa).
}

Sunto. - Si finiscono di passare in rassegna i teoremi piì importanti della teoria delle algebre a base finita, quale è esposta dallo Sconza, lasciando cadeve l'ipotesi della limitazione della base. Dei teoremi che restawo validi si dà la dimostrazione solo quand'essa è necessariamente diversa da quella dello Scorza: nel caso di teoremi la cui dimostrazione verte essenzialmente nella finitezza della base sono dati esempi di algebre per cui essi cadiono in difetto.

\section{Cap. II - Algebre senza base finita $\left({ }^{2}\right)$.}

2.1. Definizione; indipendenza dei postulati. - Dicesi algebra sul corpo $\Gamma$ un insieme $A$ di elementi, che sia un sistema nel corpo $\Gamma$, e tale che, se $(a, b)$ è una coppia ordinata di suoi elementi, sia definito un suo elemento $P(a, b)$, in modo che siano soddisfatte le seguenti proprieta:

$$
\begin{aligned}
& P(P(a, b), c)=P(a . P(b, c)) \\
& P(a, S(b, c))=S(P(a, b), P(a, c)) ; P(S(a, b), c=S(P(a, c), P(b, c)) \\
& P(D \mid \alpha, a), D(\beta, b))=D(\alpha \beta, P(a, b)), \text { se } \alpha, \beta \text { sono numeri. }
\end{aligned}
$$

Con notazioni più comuni, si può porre $P(a, b)=a b$, e $P(a, b)$ si dirà il prodotto di $a$ per $b$, mentre si riserverà il nome di prodotto scalare all'elemento $D(\alpha, \alpha)$. Le precedenti divengono allora:

$$
\begin{gathered}
(a b) c=a(b c) \\
a(b+c)=a b+a c ;(a+b \mid c=a c+b c \\
(\alpha \alpha)(\beta b)=(\alpha \beta)(a b) \text { se } \alpha, \beta \text { sono numeri. }
\end{gathered}
$$

Che l'insieme di questi postulati sia compatibile con l'insieme dei postulati (5), (6), (7), (8) è noto. Rimane da dimostrarne l'indipendenza, il che si seinde nei seguenti sottocasi:

a) la negazione di (37) è compatibile con (5), (6), (7), (8), (38), (39);

b) la negazione di (38) è compatibile con (5), (6), (7), (8), (37), (39);

c) la negazione di (39) è compatibile con (5), (6), (7), (8), (37), (38);

d) la negazione del primo dei (38) è compatibile col secondo e con (5), (6), (7), (8), (37), (39).

(1) Estratto dalla tesi di laurea (Pisa, Scuola Normale Superiore, 1942).

( $)$ La numerazione segue quella della parte I, pubblicata in questi Annali. S. IV, IF. $X X \nabla I,(1947)$, pp. 57.66 . 
Dimostrazione di a): Se $A$ è un'algebra non commutativa. in cui il prodotto si indicherà, per chiarezza, con $a \cdot b$, si ponga $a b=a \cdot b-b \cdot a$.

Si avrà allora $(a b) c=a \cdot b \cdot c-b \cdot a \cdot c-c \cdot a \cdot b+c \cdot b \cdot a ; a(b c)=a \cdot b \cdot c-$ $-a \cdot c \cdot b-b c \cdot a+c \cdot b \cdot t$, che è generalmente diverso dal precedente risultato, onde il (37) non è soddisfatto; poi : $a(b+c)=a \cdot b+a \cdot c-b \cdot a-c \cdot a$ : $a b+a c=a \cdot b-b \cdot a+a \cdot c-c \cdot a$, eguale al precedente risultato, onde il primo dei $(38)$, e analogamente il secondo, è soddisfatto: infine: $(\alpha a)(\beta b)=$ $=(\alpha \beta)(a \cdot b)-(\alpha \beta)(b \cdot a)$, e $(\alpha \beta)(a b)=(\alpha \beta)(a \cdot b)-(\alpha \beta)(b \cdot a)$, onde il $(39)$ è soddisfatto, c. v. d.

Dimostrazione di $b$ ): sia $A$ un sistema in $C(2)$ (corpo fondamentale di caratteristica 2), che abbia almeno 2 elementi distinti fra loro e dallo zero: pongasi : $a b=a$ se $b \neq 0, a b=0$ se $b=0$. 亡 allora: $(a b) c=a=a(b c)$ se $b \neq 0 \neq c ;(a 0) c=0=a \mid 0 c) ;(a b) 0=0=a(b 0)$ se $b \neq 0$, onde $A$ soddisfa il (37); poi: $(\alpha a)(\beta b)=\alpha a=(\alpha \beta)(a b)$ se $\beta b \neq 0$, ossia se $\beta=1$ e $b \neq 0 ;(\alpha a)(\beta 0)=$ $=0=(\alpha \beta)(\alpha 0) ; \alpha a)(0 b)=0=(\alpha 0)(a b)$, e quindi $A$ soddisfa al $(39)$; però $A$ non soddisfa al (38), poichè se $a, b$ sono diversi fra loro e dallo 0 , e se $c \neq 0$, è: $c(a+b)=c$, ma $c a+c b=0$.

Dimostrazione di $c$ ): sia $A$ l'insieme degli elementi $a^{\prime}$, con $a$ numero del corpo complesso $F$; pongasi : $a^{\prime}+b^{\prime}=(\alpha+b)^{\prime} ; \alpha \prime^{\prime}=(\alpha a)$ se $\alpha$ è elemento di $F$; allora $A$ è un sistema, il cui zero è $0^{\prime}$. Pongasi poi $a^{\prime} b^{\prime}=(a, b,)^{\prime}$, ove con $a_{r}$. si indica la parte reale di $a$. Allora i (37), (38) sono soddisfatti, ma non il (39), poichè se $\alpha, \beta$ sono immaginari puri diversi da 0 , ed $a, b$ reali diversi da 0 , è $:(\alpha a)(\beta b)=0$, mentre $(\alpha \beta)\left(a^{\prime} b^{\prime}\right)=(\alpha \beta a b)^{\prime}+0$. Si noti però che : (40) Se A è un sistema nel corpo $\Gamma$, per cui sono soddisfatti $i$ (37), (38), esiste un sottocorpo massimo $\mathrm{G}$ di $\Gamma$ tale che se A viene considerato in $G$, è soddisfatto anche il (39).

Dim. - Si osservi prima di tutto che il (39) è equivalente, di fronte agli altri postulati, ai due seguenti : se $a, b$ sono elementi di $A$, ed $\alpha$ è numero di $\Gamma$, ऐ̀ : $(\alpha a) b=\alpha(\alpha b) ; \alpha(\alpha b)=\alpha(\alpha b)$. 亡̀ chiaro che se $\alpha, \beta$ sono nel sotiocorpo fondamentale di $\Gamma$, il (39) è soddisfatto.

Sia allora $G_{1}$ l'insieme dei numeri $\alpha$ per cui $(\alpha a) b=\alpha(a b)$ per ogni coppia $a, b$ di elementi di $A$. Si prova facilmente che $G_{1}$ è un corpo: analogamente per il corpo $G_{z}$ degli $\alpha$ per eui $a(\alpha b)=\alpha(a b)$ per ogni coppia $a, b$; il corpo $G=G_{1} \cap G_{2}$ è quello di oui si voole dimostrare l'esistenza, c. v. d.

Dimostrazione di $d$ ): la stessa algebra definita per la dimostrazione di $b$ । dimostra anche $d$ ).

2.2 Prime definizioni; centro a algebre semplici. - Si passeranno ora in rassegna $i$ teoremi pir importanti della teoria delle algebre a base finita, quale è esposta dallo Soonza (Corpi numerici e algebre). Quelli la cui dimostrazione $\dot{\theta}$ uguale alla dimostrazione datane dallo Scckza verranno soltanto enunciati; per gli altri, verrà ciata la dimostrazione: infine, per alcuni, la 
cui dimostrazione verte essenzialmente sulla finitezza della base, saranno dati esempi di algebre per cui essi cadono in difetto.

Si ha, se $e$ è lo zero di $A: a e=e a=e$ per ogni elemento $a$ di $A$.

Infatti (12), (39), è : $a e=\alpha(0 e)=(\alpha e) 0=e$, ed analoga.

(41) Teorema. - Se a è numero di $\mathrm{\Gamma}$, ed a è elemento di $\mathrm{A}$, condizione necessaria e sufficiente affinchè sia $\alpha \mathbf{a}=0$, è che sia $\alpha=0$ ovvero $a=0$.

Se infatti $\alpha \neq 0$, ̀े $\alpha^{-1}(\alpha a)=a=0$, c. v. d.

142) Teorema. - Se $\mathrm{a}=0$, b sono elementi di $\mathrm{A}$, è $: a b=0=$ ba.

Infatti si ha $a+b=b$, onde $a b+b^{2}=b^{2}$, c. v. d. (11).

Se $a \neq 0, b \neq 0$, e $a b=0$, si dirà che $a, b$ sono rispettivamente un divi. sore sinistro o destro dello 0 , e che $a, b$ sono rispettivamente un nullifico si. nistro di $b$, e destro di $a$. Si danno anche le seguenti definizioni : se $a b=b, a$ è un modulo sinistro di $b$; analogamente per i moduli destri; se $a b=b a=b$, $a$ è un modulo di $b$; se $a \neq 0$ e $a b=b a=0, a$ è un nullifico di $b$; se $a^{2}=a \neq 0, a$ è un automodulo; se $a b=b$, ovvero $b a=b$, ovvero $a b=b a=b$ per ogni elemento $b$ di $A$, si dirà rispettivamente che $a$ è un modulo sinistro, destro, o un modulo di $A$; ed analogamente per i nullifici.

(43) Teorema. - Se a è modulo sinistro di $\mathrm{b}, e \mathrm{~b}$ modulo destro di $\mathrm{a}, \quad$ o è $\mathrm{a}=\mathrm{b}=0$, ovvero $\mathrm{a}=\mathrm{b} \grave{e}$ un automodulo.

(44) TeORena. - Un modulo destro o sinistro di un'algebra è un automodulo. (45) 'Teorema. - Un'algebra o non ha modulo, o ne ha uno solo: se ha moduli destri e moduli sinistri, ne ha uno solo, che coincide con il modulo.

(46) 'Tеовема. - Se un'algebra possiede moduli sinistri (destri) hifferenii. la differenza fra due di essi è un nullifico sinisiro (destro) dell'algebra, e da uno di essi si ottengono tutti gli altri sommandolo coi singoli mullifici sinistri (destri).

Due algebre $A, A^{\prime}$ diconsi isomorfe se i sistemi $A, A^{\prime}$ sono equivalenti, e se all'elemento $a b$, con $a, b$ in $A$, corrisponde $a^{\prime} b^{\prime}$, ove $a^{\prime}, b$ sono i corrispondenti di $a, b$ in $A^{\prime}$; diconsi reciproche, se i sistem: $A, A$ sono equivalenti, e se ad $a b$ corrisponde $b^{\prime} a^{\prime}$. Tutti i teoremi dati, e che si daranno, salle algebre. sono invarianti di fronte alle relazioni di isomorfismo a reciprocita.

Un'algebra priva di divisori dello zero dicesi primitiva. Se $B, C$ sono due sub-sistemi di $A$. sia $S$ l'insieme degli elementi di $A$ ognuno dei quali è somma di elementi del tipo $b c$, con $b$ in $B \Leftrightarrow c$ in $C: S$ è evidentemente un sistema, qualora la somma ed il prodotto scalare vi siano definiti come in $A: S$ dicesi il prodotto di $B, C$, ed indicasi con $S=B C$.

Se $S^{2} \leq S \neq 0, S$ ̀̀ evidentemente un'algebra, qualora il prodotto vi si definisca come in $A$; si dirà una sub-algebra $3 \mathrm{j} A$. e si dirà propria se $S<A$. (47) TEOREMA. - L'insiome degli elemenii di A. ognuno dei quali commuta con un dato elemento a, è una sul-algebra di $\mathrm{A}$, che dicesi l'A-commutatore di a. 1481 Teorfura. - Gli elomenti di A, ogmuno hei quali commuta con ogni ele. mento di A. formano un sistema clee. so non ì nullo, è una sub-algebrect commutatiea di $\mathrm{A}$. 
Esso dicesi la sub-algebra centrale, o il centro di $A$.

Una sub-algebra $B$ di $A$ si dirà invariante sinistra, o destra, in $A$, se $B A \leq B$, o $A B<B$ rispettivamente; si dirà invariante in $A$ se è invariante sinistra e destra.

(49) Thonfma. - Se $\mathrm{B} \neq 0$ è un sub-sistema di $\mathrm{A}$, e $\mathrm{BA} \leq \mathrm{B}(\mathrm{AB} \leq \mathrm{B}), \mathrm{B}$ è una sub-algebra invariante sinistra (destra) in A.

Una sub-algebra invariante di $A$ dicesi massima se è propria, e se non esiste nessuna sub-algebra invariante di $A$ che la contenga, senza coincidere con essa. Un'algebra dicesi semplice, se è priva di sub-algebre invarianti proprie. (50) Teorema. - Se $\mathrm{B}_{3}, \mathrm{~B}_{2}$ sono sub-algebre invarianti di $\mathrm{A}$, la $\mathrm{C}=\mathrm{B}_{4} \cap \mathrm{B}_{z}$ o è nulla, o è una sub-algebra invariante di $\mathrm{A}$. Ed ognuno dei sistemi $\mathrm{B}_{1} \mathrm{~B}_{2}$, $\mathrm{B}_{2} \mathrm{~B}_{1}$, se non è nublo, è una sub-algebra di $\mathrm{C}$ invariante in $\mathrm{A}$.

(51) Teorema. - Se a è elemento di A, l'insieme degli elementi di A $d i$ cui a $\grave{e}$ modulo sinistro (destro) costituisce un sistema che, se non è nullo, $\dot{e} u n a$ sub-algebra invariante sinistra (destra) di A.

(52) Teorema. - I nullifici destri (sinistri) di un dato elemento di A, formano un sistema che, se non è nullo, è una sub-algebra invariante sinistra (destra) di A.

2.3. Elementi singolari ed inversi. -- Un elemento $a$ di $A$ si dirà singolare sinistro, o destro, secondo che $a A<A, A a<A$; nelle algebre a base finita, ogni elemento singolare sinistro diverso da 0 è un divisore sinistro dello 0, e analogamente per il destro; ma ciò non accade per le algebre senza base finita, come si vede dalla considerazione dell'anello dei polinomi in una indeterminata sopra un corpo qualsiasi.

(53) Teorema. - Se l'elemento a dell'algebra A non è singolare sinistro (destro), per ogni b di A esiste un a di A tale che $\mathrm{ac}=\mathrm{b}$ (ca $\mathrm{b}$ ).

Un'alcebra priva di elementi singolari diversi da 0 sia sinistri che destri si dirà divisoria; essa è un gruppo rispetto all'operazione di prodotto, qualora non si tenga conto dell'elemento 0 , e quindi contiene l'elemento uniti $u$; esso è il modulo dell'algebra:

(54) Teonema. - Un'algebra divisoria è dotata di modulo.

In un'algebra col modulo $u$, un elemento $b$ dice-i inverso dell'elemetito $a$ se $a b=b a=u$. L'inverso di un elemento è unico, poichè se $a b=b a=u$, $a b^{\prime}=b^{\prime} a=u$, sarà $: b=b\left(a b^{\prime}\right)=\left(b a \mid b=b^{\prime}\right.$; l'inverso di $a$ sarà indicato con $a^{-1}$. Бว̄) Teorema. - Se a è elemento di A col modulo $\mathrm{u}, e \mathrm{~b}$, c sono tali che $\mathrm{ab}=\mathrm{ca}=\mathrm{u}, \grave{e}: \mathrm{b}=\mathrm{c}=\mathrm{a}^{-1}$.

Dim. - Infatti $c=c(a b)=(c a) b=b$.

(56) Teorema. - In un'algebra con modnlo. condizione necessaria e sufficiente affinchè un elemento sia dotato di inverso, è che esso non sia singolare nè destro, né sinistro.

Dim. - La condizione è necessaria. poichè se $a^{-1}$ esiste, e $b$ è un elemento qualsiasi, è : $b=a\left(a^{-1} b\right)$, onde $a A=A$, e analogamente per $A a$. 
Se poi $a A=A$, esiste un $b$ tale che $a b=u$, e analogamente un $c$ tale che $c a=u$, onde (55) $a$ ha inverso, c. v. d.

(57) Teorema. - Se a ha inverso, ed $\mathrm{ab}=\mathrm{u}$ (modulo), è $\mathrm{b}=\mathrm{a}^{-1}$; e analoga. mente se ba $=u$.

Infatti $b=\left(a^{-1} a\right) b=a^{-1}(a b)=a^{-1}$, c. v. d.

(58) Teorema. - Un elemento dotato di inverso non è un divisore nè destro nè sinistro dello zero.

Dim. - Se infatti fosse $a b=0$, sarebbe $b=\left(a^{-1} a\right) b=a^{-1}(a b)=0$, c. v. d.

Non è detto che ogni divisore sinistro (destro) dello 0 sia singolare sinistro (destro). Sia infatti $A$ l'algebra in $C(2)$ la cui base è formata dagli elementi $a^{\prime}$, con $a$ numero reale tale che $0 \leq a \leq 2$, col prodotto così definito:

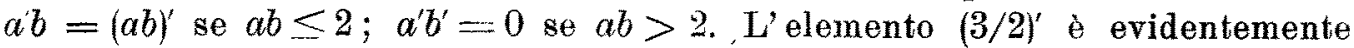
un divisore sinistro dello 0 , ma $\grave{\text { eे }}(3 / 2)^{\prime} A=A$, poichè esiste sempre il numero $x$ compreso fra 0 e 2 tale che $(3 / 2) x=a$ se $0 \leq a \leq 2$. Però :

(59) Teorema. - Un'algebra divisoria è primitiva.

Essa, infatti, ha (5a) il modnlo $u$, ed ogni elemento $\neq 0$ è dotato di in. verso e quindi, per la (58), tale algebra è priva di divisori sinistri o destri dello 0 , c. v. d.

Possono invece aversi algebre primitive senza modulo, e non divisorie: tale è infatti l'algebra sul corpo $\Gamma$ formata dai polinomi di grado maggiore od eguale ad 1, in una indeterminata $e$ con coefficienti in $\Gamma$.

(60) TEOREMa. - Condizione necessaria e sufficiente affinchè un'algebra abbia qualche modulo sinistro (destro), è che contenga qualche elemento che non sia nè singolare sinistro (destro), nè divisore sinistro (destro) dello 0.

Dim. - La condizione è evidentemente necessaria. Se poi $a$ è un tale elemento, esiste un $u$ per cui $a u=a:$ se $b$ è elemento di $A$, ̀े $a u b=a b$, onde $u b=b$, c. v. d.

(61) Teorema. - Ogni elemento di una sub-algebra invariante propria sinistra (destra) in A è singolare sinistro (destro) in A.

È evidente; da (56) si ha poi :

(62) Thorema. - Un' algebra divisoria è semplice.

(63) Thorema. - Una sub-algebra di un'algebra primitiva è primitiva.

2.4. Elementi pseudonnlli. -- Sia $A$ un'algebra: si avrà $A \geq A^{2} \geq A^{3} \geq \ldots$. Può essere che vi sia un intero $r$ thle ohe $A^{r}=A^{r+1}$, ed $A^{r-1}>A^{r}$; tale $r$ si dirà l'indice di $A$. $A^{\prime \prime}$ dicesi il nucleo di $A$, e se esso è nullo, $A$ dicesi psendonulla; un'algebra pseudonulla di indice 2 dicesi zero-algebra.

(64) TEonema. - So A è pseudonulla di indice r, ogni sua sub-algebra ̀̀ pseudonulla di indice minore od uguale ad $\mathrm{r}$.

(65) TEorfma. - Una zero-algebra è semplice se, e solo se è di ordine uno. (66) Teorema. - Il nucleo di un'algebra, se non è nullo, è una sub-algebra invariante dell'algebra data. Th' algebra semplice è di indice uno o due, e nel secondo caso è una zero-algebra di ordine uno. 
Un'algebra si dirà semisemplice se è priva di sub-algebre invarianti pseudonulle.

(67) Teonema. - Un'algebra semisemplice, che non sia una zero-algebra di ordine uno, inon è pseudonulla. Se A ha dei nullifici, essi, insieme allo 0, costituiscono una zero-algebra invariante in A. Un'algebra semisemplice, che non sia una zero-algebra di ordine uno, è priva di nullifici.

Un elemento $a \neq 0$ di un'algebra dicesi pseudonullo se qualche sua potenza è nulla; ed il massimo intero $r$ per oui $a^{r} \neq 0$ dicesi il grado di $a$. (68) Teorema. - Se un'algebra ha elementi pseudonulli, ne ha certamente di grado uno.

(69) Teoriira. - Se ab è nullo, o pseudonullo, tale è ba, e $i$ loro gradi differiscono al più di uno.

(70) Teorema. - Ogni elemento dẹ un'algebra pseudonulla di indice r, è pseudonullo di grado minore" di $\mathrm{r}$.

Non è però vero, come nelle algebre a base finita, il reciproco; si consideri infatti l'algebra $A$ in $C(2)$ che ha per base l'insieme degli elementi $a,(r=1,2, \ldots)$ in cui il prodotto è così definito: $a, a_{s}=a_{r s}$ se $r, s$ sono primi fra loro; $a_{r} a_{s}=0$ negli altri casi. Il numero 1 non si considererà primo con nessun numero. È chiaro che tale algebra soddisfa a (37), e che in essa ogni elemento è pseudonullo di grado al più eguale al numero di elementi della base di oni esso è somma; però non è pseudonulla, perchè si può avere an prodotto di un numero qualsiasi di elementi che è diverso da 0 ; esso è infatti il prodotto $a_{r_{1}} a_{r_{2}} \ldots a_{r_{n}}$, ove $r_{1}, r_{2}, \ldots, r_{n}$ sono i primi $n$ numeri primi.

2.5. Sonma diretta; differenza di algkbre - Se $S_{i}, S_{j}$ sono sub-sistemi di un'algebra $A$, la loro somma si dirà dirella se essi sono disgiunti, e se $S_{i} S_{j} \cdots S_{j} S_{i}=0$. Un'algebra dicesi riducilile o irriducibile secondochè pnò pensarsi, o no, come somma diretra di sue sub-algebre. Non ̀̀ detto che un'algrbra riducibile sia scomponibile nella somma di algebre irriducibili, come accade sempre per le algebre a hase finita; basta pensare ad una zeroalgebra senza base finita.

(71) Thorema. - Un'algebra riducibile is sommatoria se, e solo se tali sono le algebre di cui essa è somma direttu.

Deriva immediatamente da (18), (19).

(72) THorwa. - Lindice di $\mathrm{S}=\mathrm{S}_{1}+\ldots ; \mathrm{S}_{\mathrm{n}}\left({ }^{3}\right)$ esiste se, e solo se ogni $\mathrm{S}_{\mathrm{i}}$ ha indice; e l'indice di $\mathrm{s}$ è eguale cll'indice di quell's che ha indice massimo. (73) Teorema. $-\mathrm{S}=\mathrm{S}_{1}+\ldots+\mathrm{S}_{\mathrm{n}} \dot{e}$, pseudomulla se, e solo se tale è ogni $\mathrm{S}_{\mathrm{i}}$. 174) Teorext - Se $\mathrm{S}=\mathrm{S}_{1}+\ldots+\mathrm{S}_{\mathrm{n}}$, ogni $\mathrm{S}_{\mathrm{i}}$, se è un'algebra, è una subalgebra invariante propria di $\mathrm{S}$. Un'algebra semplice è irriducibile,

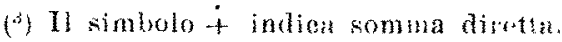


Ed anche:

(75) Thorema. - Se $\mathrm{S}_{1}, \ldots, \mathrm{S}_{\mathbf{n}}$ sono sub-algebre invarianti e complementari in $\mathrm{A}, \grave{e}: \mathrm{A}=\mathrm{S}_{1}+\ldots+\mathrm{S}_{\mathbf{n}}$.

In quel che segue, supporremo $a, b$ elementi di $A=A_{1}+\ldots+A_{n}$, con $A_{1}, \ldots A_{n}$ algebre, e $a=\sum_{1}^{n} a_{i}, b=\sum_{i}^{n} b_{i}$, con $a_{i}, b_{i}$ elementi di $A_{i}$.

(76) Teorema. - Se a è un modulo sinistro (destro) di b, tale è $\mathrm{a}_{\mathrm{i}}$ per $\mathrm{b}_{\mathrm{i}}$, e reciprocamente.

(77) Teonema. - L'algebra $\mathrm{A}=\mathrm{A}_{1}+\ldots+\mathrm{A}_{\mathrm{n}}$ è dotata di moduli sinistri (destri) se, e solo se lo sono le $\mathrm{A}_{1}, \ldots, \mathrm{A}_{\mathrm{n}}$; e tali moduli sono tutti e soli gli elementi $\mathrm{u}=\mathrm{u}_{1}+\ldots \mathrm{u}_{\mathrm{n}}$, ove $\mathrm{u}_{\mathrm{i}}$ è modulo sinistro (destro) di $\mathrm{A}_{\mathrm{i}}$. Lo stesso dicasi pei nullifici sinistri (destri).

(78) Teorema. - Colle notazioni di (77), la A ha centro se, e solo se lo ha almeno una delle $\mathrm{A}_{\mathbf{i}}$; e tale centro è la somma diretta dei centri di quelle $\mathrm{A}_{\mathbf{i}}$ ohe hanno centro.

(79) Teorema. - L' 'lemento a è pseudonullo se, e solo se gli $a_{1}$ non sono tutti nulli, e se quelli fra essi che sono diversi da 0 sono pseudonulli. Il grado di a $\grave{e}$ il grado di quello fra gli $a_{\mathrm{i}}$ che ha grado massimo. L'elemento a è un automodulo se. e solo se gli a, non sono tutti nulli. e se quelli, fra essi, che sono diversi da 0 sono automoduli.

La teoria della congruenza rispetto ad un sistema si svolge come per le algebre a base finita, e vale ancora il

(80) Teorema di Molien. - Se B è unc sub-algebra invariante propria di A, si può definive il prodotto fra le classi di. $\mathrm{A}(\bmod \mathrm{B})$ in modo che esse formino un'algebra, che si dirà la differenza fra $\mathrm{A} e \mathrm{~B}$, e si inducherà con $\mathrm{A}-\mathrm{B}$. (81) Teorena. - Se A $=\mathrm{B}+\mathrm{C}$, con B, C algebre, B è isomorfa ad A - C,e $\mathrm{C}$ ad $\mathrm{A}-\mathrm{B}$.

(8) Teonema. - Se B è una sub-algebra del sistema sommatorio A, A - B è sommatorio.

Dim. - Sia infatti $C$ un complementare di $B$ in $A$; ogni classe di $A$ $(\bmod B)$ contiene un solo elemento di $C$, e ogni elemento di $C$ è contenuto in una di tali classi; inoltre, $C$ è equivalente ad $A-B$, onde (19) $A-B$ è sommatorio, c. v. d.

(83) Teorema. - Se A, B sono algebre nel corpo $\mathrm{P}$, esiste un'algebra $\mathrm{C}$ che è somma diretta di due algebre isomorfe ad A, B rispetivamente.

Dim. - Sia $C$ l'algebra formata dalle coppie $(a, b)$, con $a$ elemento di $A$, e $b$ di $B$, e colle operazioni cosi definite:

$$
(a, b)+\left(a^{\prime}, b^{\prime}\right)=\left(a+a^{\prime}, b+b^{\prime}\right) ;(a, b)\left(a^{\prime}, b^{\prime}\right)=\left(a a^{\prime}, b b^{\prime}\right)
$$

$\alpha(a, b)=(\alpha a, \alpha b)$ se $\alpha$ è numero di $\Gamma$. 亡̀ facile constatare che $C$ è effettivamente un'algebra; e i sub-sistemi formati dagli elementi del tipo $(a, 0),(0, b)$ sono algebre isomorfe ad $A, B$ rispettivamente, e $C$ è la loro somma diretta c. v. d. 
2.6. Prodotto diretto. - Se $B, C$ sono sistemi non nulli dell'algebra $A$, tali che ogni elemento di di $B$ commuti con ogni elemento di $C$, gli elementi $b_{1}, \ldots, b_{n}$ di $B$ si diranno indipendenti in $C$, se può essere $c_{1} b_{1}+\ldots+c_{n} b_{n}=0$, con $c_{1}, \ldots, c_{n}$ elementi di $C$, quando, e solo quando sia

$$
c_{1}=\ldots=c_{n}=0 \text {. }
$$

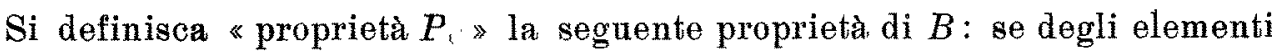
di $B$ sono dipendenti in $C$, essi sono fra loro dipendenti: e analogamente per la proprietà $P_{B}$ di $C$.

(84) Lemma. - Se $\mathrm{B}$ gode di $\mathrm{P}_{\mathrm{C}}$, allora $\mathrm{O}$ gode di $\mathrm{P}_{\mathrm{B}}$.

Dim. - Siano $c_{1}, \ldots, c_{n}$ elementi di $C$, tutti diversi da 0 , dipendenti in $B$ : $b_{1} c_{1}+\ldots+b_{n} c_{n}=0$, ove gli elementi $b_{1}, \ldots, b_{n}$ possono supporsi tutti non nulli; poichè $B$ gode di $P_{C}$, esistono dei numeri $\alpha_{1}, \ldots, \alpha_{n}$ non tutti nulli per cui $\alpha_{1} b_{1}+\ldots+\alpha_{n} b_{n}=0$; supposto $\alpha_{1} \neq 0$, è : $b_{1}=\alpha_{2}^{\prime} b_{2}+\ldots+\alpha_{11}{ }^{\prime} b_{n}$, onde $\alpha_{2}^{\prime} b_{2} c_{1}+\ldots+\alpha_{n}{ }^{\prime} b_{n} c_{1}+b_{2} c_{2}+\ldots+b_{n} c_{n}=\left(\alpha_{2}^{\prime} c_{1}+c_{2}\right) b_{2}+\ldots+\left(\alpha_{n}{ }^{\prime} c_{1}+c_{n}\right)^{b_{n}}=0$. Se in quest' espressione i coefficienti delle $b_{2}, \ldots, b_{n}$ non sono tutti $\neq 0$, le $c_{1}, \ldots, c_{n}$ sono dipendenti, che è cio che si vuole dimostrare; altrimenti si ripete il ragionamento, e cosi proseguendo; se non si arrivasse ad un punto in cui uno dei coefficienti delle $b_{n}, b_{r+1}, \ldots, b_{n}$ è nullo, si arriverebbe ad avere una espressione della forma $\left(\beta_{1} c_{1}+\beta_{2} c_{2}+\ldots+\beta_{n-1} c_{n-1}+c_{n}\right) b_{n}=0$, con $\beta_{1}, \ldots, \beta_{n-1}$ numeri; ed allora sarebbe $\beta_{1} c_{1}+\ldots+\beta_{n-1} c_{n-1}+c_{n}=0$, c. v. d.

Se allora $B, C$ sono sub-sistemi di $A$, il loro prodotto $D=B C$ dicesi diretto ed indicasi con $B \times C=C \times B$ se: ogni elemento di $B$ commuta con ogni elemento di $C ; B$ gode di $P_{C}$ o, il che è $(84)$ lo stesso, $C$ gode di $P_{B}$. E facile vedere che questa definizione di prodotto diretto coincide, nel caso di algebre a base finita, con quella che si dà comunemente.

(85) Teonema. - Se $\mathrm{D}=\mathrm{B} \times \mathrm{C}$, con $\mathrm{B}$, $\mathrm{C}$ sub-clyebre di $\mathrm{A}$, coi moduli b, a rispettivamente, $\mathrm{D}$ è prodotto diretto di due algebre con lo stesso modulo, isomorfe a B, C.

Dim. - Si considerino infatti le algebre $b C$ e $c B$; esse hanno il modulo $b c$, e se $D^{\prime}=(b C)(c B)$, ogni elemento di $D^{\prime}$ è della forma $d^{\prime}=b c b_{1} c_{1}+\ldots+b c b_{n} c_{n}$, con $b_{1}, \ldots . b_{n}$ elementi di $B, c_{1}, \ldots, c_{n}$ elementi di $C$ : percio $d^{\prime}=b_{1} c_{1}+\ldots+b_{n}, c_{n}$ è elemento di $D$, e viceversa, onde $D^{\prime}=D$. Il resto è immediato, c. v. d.

(86) Teorema. - Ogni algebra è isomorfa ad una sub-algebra invariante di un'algebra con modulo.

Dim. - Sia $A$ un'algebra priva di modulo, e si consideri l'insieme delle coppie $(\alpha, \alpha)$ con $a$ elemento di $A$ ed $\alpha$ numero di $\Gamma$; pongasi $\beta(\alpha, \alpha)=(\beta \alpha, \alpha \beta)$; $(a, \alpha)+(b, \beta)=(a+b, \alpha+\beta) ;(a, \alpha)(b, \beta)=(\alpha b+\alpha b+\beta a, \alpha \beta)$. Si constata immediatamente ohe l'insieme $K$ così definito è un'algebra col modulo $(0,1)$, e che in essa l'insieme deglị elementi $\langle a, 0)$ è una sub-algebra isomorfa aद $A$; che poi tale sub-algebra sia invariante in $K$ è evidente, c. v. d.

(87) Thonema. - Se A, B sono due algebre sul corpo $\Gamma$, esiste un'algebra $\mathrm{C}$ nel corpo $\Gamma$ che è prodotto diretto di due algebre $\mathrm{A}^{\prime}, \mathrm{B}^{\prime}$ isomorfe ad $\mathrm{A}, \mathrm{B}$ rispeltiramente. 
Dim. - Sia $L$ l'insieme degli elementi $\left(\begin{array}{l}a_{1}, \ldots, a_{r} \\ b_{1}, \ldots, b_{r}\end{array}\right)(r=1,2, \ldots)$, con $a_{i}$ elemento di $A, b_{i}$ elemento di $B$; si indichera, usando il simbolismo delle matrici, con a l'n-complesso orizzontale $\left(a_{1}, \ldots, a_{n}\right)$, e con $\left(\begin{array}{l}a \\ b\end{array}\right)$ l'elemento $\left(\begin{array}{l}a_{1}, \ldots, a_{v} \\ b_{1}, \ldots, b,\end{array}\right)$ di $L$. L'elemento $b$ si chiamerà il corrispondente di $a$.

(88) Lemara. - Se $\left(\begin{array}{l}a \\ b\end{array}\right)$ è elemento di L, e $a_{1}, a_{1}{ }^{\prime}$ sono insiemi di elementi indipendenti di a, tali che ogni elemento di a sia loro combinazione, e, chiamati $\mathrm{a}_{2}, \mathrm{a}_{2}{ }^{\prime} i$ rimanenti elementi di a, ordinati in modo qualsiasi, si ha $\mathrm{a}_{2}=\mathrm{a}_{1} \mathrm{~A}$, $\mathrm{a}_{2}{ }^{\prime}=\mathrm{a}_{1}{ }^{\prime} \mathrm{A}^{\prime}$. con $\mathrm{A}$. $\mathrm{A}^{\prime}$ matrici in $\mathrm{I}$, pongasi: $\mathrm{b}_{3}=\mathrm{b}_{1}+\mathrm{b}_{2} \mathrm{~A}_{-1} ; \mathrm{b}_{3}{ }^{\prime}=\mathrm{b}_{1}{ }^{\prime}+\mathrm{B}_{2}{ }^{\prime} \mathrm{A}^{\prime}{ }_{-1}$, dove $\mathrm{b}_{1}, \mathrm{~b}_{2}, \mathrm{~b}_{1}{ }^{\prime}, \mathrm{b}_{2}^{\prime}$ sono $i$ corrispondenti di $\mathrm{a}_{1} . \mathrm{a}_{2}, \mathrm{a}_{1}^{\prime}, \mathrm{a}_{2}^{\prime}$; allora $\mathrm{b}_{3}=\mathrm{b}_{3}{ }^{\prime} \mathrm{S}_{-1}$, dove $\mathrm{S}$ è matrice tale che $\mathrm{a}_{1}^{\prime}=\mathrm{a}_{1} \mathrm{~S}$.

Dim. - Sia $K$ matrice i cui elementi sono soltanto 0 ed 1 , tale che $\left(a_{1}^{\prime} \mid a_{0}{ }^{\prime}\right)=\left(a_{1} \mid a_{2}\right) K$, e perciò $\left(b_{1}^{\prime} \mid b_{2}^{\prime}\right)=\left(b_{1} \mid b_{2}\right) K$; si ha $a_{1}(I \mid A)=\left(a_{1} \mid a_{2}\right)$, ove $I$ è matrice identica di ordine opportuno; ed è anche : $a_{1} S\left(I \mid A^{\prime}\right) K_{-1}=$ $=a_{1}\left(S \mid S A^{\prime}\right) K_{-1}=\left(a_{1}^{\prime} \mid a_{2}\right) K_{-1}=\left(a_{1}^{\prime} \mid a_{2}{ }^{\prime}\right) K^{-1}=\left(a_{1} \mid a_{0}\right)$, attesa l'ortogonalità di $K$; perciò, essendo gli elementi di $a$ indipendenti, è $(I \mid A)=S\left(I \mid A^{\prime}\right) K_{-1}$, ed allora $\left(\frac{I}{A_{-1}}\right)=K\left(\begin{array}{c}I \\ A_{-1}^{\prime}\end{array}\right) S_{-1}$, e $\left(b_{1} \mid b_{2}\right)\left(\frac{I}{A_{-1}}\right)=b_{1}+b_{2} A_{-1}=b_{3}$; $\left(b_{1} \mid b_{2}\right) K\left(\begin{array}{c}I \\ A_{-1}^{\prime}\end{array}\right) S_{-1}=\left(b_{1}^{\prime} \mid b_{2}^{\prime}\right)\left(\frac{I}{A_{-1}^{\prime}}\right) S_{-1}=\left(b_{1}^{\prime}+b_{2}^{\prime} A_{-1}^{\prime}\right) S_{-1}=b_{3} S_{-1}^{\prime}$, onde $b_{3}=b_{3}{ }^{\prime} S_{-1}$, ed il lemma è dimostrato.

Il lemma vale, naturalmente, anche scambiando i ruoli delle $a$ e delle $b$. Perciò, dato un elemento qualsiasi $\left(\begin{array}{l}a \\ b\end{array}\right)$ di $L$, si può costruire un elemento $\left(\begin{array}{l}a^{\prime} \\ b^{\prime}\end{array}\right)$ con $a^{\prime}$ formato tutto di elementi indipendenti di $a$, e tali che ogni elemento di $a$ sia loro combinazione, e con $b^{\prime}$ costruito come è indicato nel lemma per: costruire $b_{3}$; e per il lemma stesso, si possono ripartire gli elementi di $L$ in classi, ponendo due elementi $\left(\begin{array}{l}a \\ b\end{array}\right),\left(\begin{array}{l}a^{\prime} \\ b^{\prime}\end{array}\right)$ nella stessa classe, se essendo i complessi $a, a^{\prime}$ formati ciascuno da elementi indipendenti, esiste una matrice $S$ per cui $a^{\prime}=a S$, e $b=b^{\prime} S_{-1}$, o se, non essendo verificata quell' ipotesi, $\left(\begin{array}{l}a \\ b\end{array}\right)$ e $\left(\begin{array}{l}a^{\prime} \\ b^{\prime}\end{array}\right)$ sono riducibili, nel modo indicato in (88) per ottenere $\left(\begin{array}{l}a_{1} \\ b_{3}\end{array}\right)$, ad elementi $\left(\begin{array}{l}a_{1} \\ b_{1}\end{array}\right),\left(\begin{array}{l}a_{1}^{\prime} \\ b_{1}^{\prime}\end{array}\right)$ con le $a_{1}, a_{1}^{\prime}$ soddisfacenti a quell'ipotesi, e tali che esi. sta la matrice $S$ per cui $b_{1}=b_{1}{ }^{\prime} S_{-1} e_{2} a_{1}{ }^{\prime}=a_{1} S$. Il lemma assicura che ogni elemento appartiene ad una sola classe. Si indicherà con $\left[\begin{array}{l}a \\ b\end{array}\right]$ la classe che contiene $\left(\begin{array}{l}a \\ b\end{array}\right)$. Pongasi : $x\left[\begin{array}{l}a \\ b\end{array}\right]=\left[\begin{array}{l}a \\ x b\end{array}\right]$, se $x$ è numero di $\Gamma$; si deve dimostrare 
che questa definizione è indipendente dagli elementi di $L$ scelti per deter* minare le classi; se infatti $\left[\begin{array}{l}a \\ b\end{array}\right]=\left[\begin{array}{l}c \\ d\end{array}\right]$, sia $\left[\begin{array}{l}a^{\prime} \\ b^{\prime}\end{array}\right]=\left[\begin{array}{l}a \\ b\end{array}\right]$, con $a^{\prime}$ formato da elementi indipendenti ; allora è $\left[\begin{array}{l}a^{\prime} \\ x b\end{array}\right]=\left[\begin{array}{l}a \\ x b\end{array}\right]=\left[\begin{array}{l}c \\ x d\end{array}\right]$, onde $x\left[\begin{array}{l}a \\ b\end{array}\right]=x\left[\begin{array}{l}c \\ d\end{array}\right]$.

Si definisca la somma fra due classi di $L$ come segue: se $\left[\begin{array}{l}a \\ b\end{array}\right],\left[\begin{array}{l}c \\ d\end{array}\right]$ sono classi, con $a, b, c, d$ complessi orizzontali, indicati con $A, C$ i sistemi formati dalle combinazioni degli elementi di $a$ e di $c$ rispettivamente, sia $D=A \cap C$; sia $h^{\prime}$ una base di $D, h$ una base di un complementare di $D$ in $A$, $h^{\prime \prime}$ una base di un complementare di $D$ in $O$ : esisteranno i $k, k^{\prime}, k^{\prime \prime}, k_{1}^{\prime}$ tali che:

$$
\left[\begin{array}{l}
a \\
b
\end{array}\right]=\left[\begin{array}{ll}
h, & h^{\prime} \\
k, & k^{\prime}
\end{array}\right],\left[\begin{array}{l}
c \\
d
\end{array}\right]=\left[\begin{array}{ll}
h^{\prime}, & h^{\prime \prime} \\
k_{1}^{\prime}, & k^{\prime \prime}
\end{array}\right]
$$

pongasi

$$
\left[\begin{array}{l}
a \\
b
\end{array}\right]+\left[\begin{array}{l}
c \\
d
\end{array}\right]=\left[\begin{array}{lcc}
h, & h^{\prime}, & h^{\prime \prime} \\
k, & k^{\prime}+k_{i}^{\prime}, & k^{\prime \prime}
\end{array}\right]
$$

si dimostra che questa definizione è indipendente dagli $\left(\begin{array}{l}a \\ b\end{array}\right),\left(\begin{array}{l}c \\ d\end{array}\right)$ scelli per identificare le classi. Se infatti si scegliessero altri elementi, $A, C, D$ rimar. rebbero invariati, e le basi $h, h^{\prime}, \bar{h}^{\prime \prime}$ godrebbero delle stesse proprietà di cui godono $h, h^{\prime}, h^{\prime \prime}$, e si avrebbero dei corrispondenti elementi $\bar{k}, \bar{k}^{\prime}, \ddot{k}^{\prime \prime}, k^{\prime}$. Si avrebbe allora:

$$
\begin{cases}\left(\bar{h} \mid \bar{h}^{\prime}\right)=\left(h \mid h^{\prime}\right) S & \left(\bar{h}^{\prime} \mid h^{\prime \prime}\right)=\left(h^{\prime} \mid h^{\prime \prime}\right) T \\ \left(k \mid k^{\prime}\right)=\left(\bar{k} \mid \bar{k}^{\prime}\right) S_{-1} & \left(k_{1}^{\prime} \mid k^{\prime \prime}\right)=\left(\bar{k}_{1}^{\prime} \mid \bar{k}_{1}^{\prime \prime}\right) T_{-i}\end{cases}
$$

ed $S, T$ dovrebbero trasformare $h^{\prime}$ in $\bar{h}^{\prime}$ per qualsiasi $k, k^{\prime \prime}$; percio :

$$
S=\left(\frac{S^{\prime}}{S^{\prime \prime}} \mid \frac{0}{K}\right) ; \quad T=\left(\frac{K}{0} \mid \frac{T^{\prime \prime}}{T^{\prime \prime}}\right) ;
$$

si deve dimostrare che so $\left(h\left|\bar{h}^{\prime}\right| \bar{h}^{\prime \prime}\right)=\left(h\left|h^{\prime}\right| h^{\prime \prime}\right) R$, allora $\left(k_{i}\left|k^{\prime}+k_{1}^{\prime}\right| k^{\prime \prime}\right)=$ $=\left(\vec{k} \mid \vec{k}^{\prime}+\bar{k}_{1}{ }^{\prime} ; k^{\prime \prime}\right) R_{-1}$.

Ora, in effetti, $\dot{e}$

$$
R=\left(\begin{array}{c|c|c}
S^{\prime \prime \prime} & \frac{0}{K} & \frac{0}{S^{\prime}} \\
\hline 0 & 0 & \frac{T^{\prime \prime}}{T^{\prime \prime}}
\end{array}\right)
$$

e per lo $(89)$ si vede che la relazione è verificata. Ogni elemento $\left[\begin{array}{l}p \\ q\end{array}\right]$ si può esprimer: come somma di elementi del tipo $\left[\begin{array}{l}a \\ b\end{array}\right]$, con $a, b$ elementi, e non complessi orizzontali. Non è detto che cio possa avvenire in un sol modo. 
(90) Lемма. - Condizione sufficiente affinchè sia $\left[\begin{array}{l}\mathrm{a} \\ \mathrm{b}\end{array}\right]=\left[\begin{array}{l}\mathrm{c} \\ \mathrm{d}\end{array}\right]$, è che esista una matrice $\mathrm{S}$ tale che $\mathrm{c}=\mathrm{aS}, \mathrm{b}=\mathrm{dS}_{-1} ;$ se a $\grave{e}$ formato di elementi fra loro indipendenti, la condizione $\dot{e}$ anche necessaria.

Dim. - Sia infatti $a=\left(a_{1} \mid a_{2}\right), b=\left(b_{1} \mid b_{2}\right), c=\left(c_{1} \mid c_{2}\right), d=\left(d_{1} \mid d_{2}\right)$, con $a_{1}$ e $c_{1}$ formati da elementi indipendenti, e tali che $a_{2}=a_{1} T, c_{3}=c_{1} R$. Posto: $b_{3}=b_{1}+b_{2} T_{-1} ; d_{3}=d_{1}+d_{2} R_{-1}$, sarà $(88):\left[\begin{array}{l}a \\ b\end{array}\right]=\left[\begin{array}{l}a_{1} \\ b_{3}\end{array}\right],\left[\begin{array}{l}c \\ d\end{array}\right]=\left[\begin{array}{l}c_{1} \\ d_{3}\end{array}\right] ;$ si deve dimostrare che esiste una matrice $U$ tale che $c_{1}=a_{1} U, b_{3}=d_{2} U_{-1}$; ora, se $c=a S$, la $U$ per cui $c_{1}=a_{1} U$ esiste certamente, ed è : $a_{1}(I \mid T) S=$ $=\left(a_{1} \mid a_{2}\right) S=\left(c_{1} \mid c_{2}\right)=c, a_{1} U(I \mid R)=c_{1}(I \mid R)=\left(c_{1} \mid c_{2}\right)=c$, con $I$ matrice identica di ordine opportuno. Perció, attesa l'indipendenza degli elementi di $a_{1}$, ̀̀ $(I \mid T) S=U(I \mid R)$, onde

$$
S_{-1}\left(\frac{I}{T_{-1}}\right)=\left(\frac{I}{R_{-1}}\right) U_{-1}
$$

ed allora :

$b_{3}=b_{1}+b_{2} T_{-1}=b\left(\frac{I}{T_{-1}^{-1}}\right)=d S_{-1}\left(\frac{I}{T_{-1}}\right)=d\left(\begin{array}{c}I \\ R_{-1}\end{array}\right) U_{-?}=d_{1} U_{-1}+d_{2} R_{-1} U_{-1}=d_{3} U_{-3}$.

$\grave{E}$ così dimostrata la sufficienza delle condizione. Sia ora a formata da elementi indipendenti; allora certo esiste una $S$ per cui $c=a S$, e se $c_{2}=c_{1} R$, posto $d_{3}=d_{1}+d_{2} R_{-1}$, esisterà una $T$ tale che $c_{1}=a T$, e $b=d_{3} T_{-1}=$ $=\left(d_{1} \mid d_{2}\right)\left(\begin{array}{c}I \\ R_{-1}\end{array}\right) T_{-1}$. Sarà inoltre: $c=\left(c_{1} \mid c_{2}\right)=a(T, T R)=a T(I \mid R)$, e perciò $S=T(I \mid R)$; ed allora $d S_{-1}=\left(d_{1} \mid d_{2}\right)\left(\begin{array}{c}I \\ R_{--1}\end{array}\right) T_{-1}=\left(d_{1}+d_{2} R_{-1}\right) T_{-1}=$ $=d_{3} T_{-i}=b$, c. v. d. Si ha poi :

$$
\left[\begin{array}{l}
a \\
b
\end{array}\right]+\left[\begin{array}{l}
c \\
d
\end{array}\right]=\left[\begin{array}{ll}
a, & c \\
b, & d
\end{array}\right]
$$

Dim. - Siano $h, h^{\prime}, h^{\prime \prime}, k, k^{\prime}, k^{\prime \prime}, k_{1}^{\prime}$ i complessi usati per la definizione di $\left[\begin{array}{l}a \\ b\end{array}\right]+\left[\begin{array}{l}c \\ d\end{array}\right] ;$ sarà $(90)$

$$
\begin{array}{ll}
a=\left(h \quad h^{\prime}\right) S & c=\left(h^{\prime} \mid h^{\prime \prime}\right) T \\
\left(k \mid k^{\prime}\right)=b S_{-1} & \left(k_{1}^{\prime} \mid k^{\prime \prime}\right)=d T_{-1},
\end{array}
$$

e, posto $S=\left(\frac{S^{\prime}}{S^{\prime \prime}}\right) \cdot T=\left(\frac{T^{\prime}}{T^{\prime \prime}}\right)$, con $S^{\prime \prime}, T^{\prime}$ matrici di tante righe, quante sono le colonne di $h, h^{\prime}$ rispettivamente sarà :

$$
\begin{array}{ll}
a=h S^{\prime}+h^{\prime} S^{\prime \prime} & e=h^{\prime} T^{\prime}+h^{\prime \prime} T^{\prime \prime} \\
\left(k \mid k^{\prime}\right)=b\left(S_{-1}^{\prime} \mid S^{\prime \prime}\right) & \left(k_{1}^{\prime} \mid k^{\prime \prime}\right)=d\left(T_{-1}^{\prime} \mid T_{-1}^{\prime \prime}\right) ;
\end{array}
$$


infine:

$$
(a \mid c)=\left(h, h^{\prime} \mid h^{\prime \prime}\right)\left(\frac{\frac{S^{\prime}}{S^{\prime \prime}}}{\frac{0}{0}} \mid \frac{0}{T^{\prime \prime}}\right) ;
$$

poichè $\left[\begin{array}{l}a \\ b\end{array}\right]+\left[\begin{array}{l}c \\ d\end{array}\right]=\left[\begin{array}{ccc}h, & h^{\prime}, & h^{\prime \prime} \\ k, & k^{\prime}+k_{\imath}{ }^{\prime}, & k^{\prime \prime}\end{array}\right]$, basta dimostrare (90) che $\left(k\left|k^{\prime}+k_{1}{ }^{\prime}\right| k^{\prime \prime}\right)=$ $=(b \mid d)\left(\frac{S_{-1}^{\prime}}{0}\left|\frac{S^{\prime \prime}-1}{T_{-}^{\prime}}\right| \frac{0}{T^{\prime \prime}}\right)$ per aver dimostrato l'asserto; ora è effettivamente $(b \mid d)\left(\frac{S_{-1}^{\prime}}{0}\left|\begin{array}{c|c}S_{-1}^{\prime \prime} & 0 \\ T_{-1}^{\prime}\end{array}\right| T_{-1}^{\prime \prime}\right)=\left(b S_{-1}^{\prime} \mid b{S^{\prime \prime}}_{-1}^{\prime}+d T_{-1}^{\prime} \quad d T_{-1}^{\prime \prime}\right)=\left(k\left|k^{\prime}+k_{1}^{\prime}\right| k^{\prime \prime}\right)$, c. v. d.

Con le operazioni cosi definite, si constata che l'insieme $J$ delle classi di $L$ è un sistema, perchè soddisfa alla $(5),(6),(7),(8)$.

Se $a_{1}, \ldots, a_{n}, b_{1}, \ldots, b_{n}, c_{1}, \ldots, c_{n}, d_{1}, \ldots d_{n}$ sono ora elementi, e non complessi orizzontali, pongasi :

$$
\left[\begin{array}{l}
a_{1}, \ldots, a_{v} \\
b_{1}, \ldots, b_{r}
\end{array}\right]\left[\begin{array}{l}
c_{1}, \ldots, c_{n} \\
d_{1}, \ldots, d_{n}
\end{array}\right]=\left[\begin{array}{l}
a_{1} c_{1}, \ldots, a_{1} c_{n}, \ldots, a_{r} c_{1}, \ldots, a_{2} \cdot c_{n} \\
b_{1} d_{1}, \ldots, b_{1} d_{n}, \ldots, b_{r} d_{1}, \ldots, b, d . .
\end{array}\right]
$$

si vuole dimostrare che questa definizione è indipendente dagli elementi $\left(\begin{array}{l}a_{1}, \ldots, a_{r} \\ b_{1}, \ldots, b\end{array}\right),\left(\begin{array}{l}c_{1}, \ldots, c_{r} \\ d_{1}, \ldots, d_{r}\end{array}\right)$ usati per definire la classi. Basterà dimostrare che: se $c_{1}^{\prime}, \ldots c_{m}^{\prime}$ sono indipendenti, ed ogni $c_{i}$ è loro combinazione, e se $\left[\begin{array}{l}c_{1}^{\prime}, \ldots, c_{m}^{\prime} \\ d_{1}^{\prime}, \ldots, d_{m}^{\prime}\end{array}\right]=\left[\begin{array}{l}c_{1}, \ldots, c_{n} \\ d_{1}, \ldots, d_{n}\end{array}\right]$, allora

$$
\left[\begin{array}{l}
a_{1} c_{1}^{\prime}, \ldots, a_{1} c^{\prime}{ }_{m}, \ldots, a_{r} c_{1}^{\prime}, \ldots, a_{r} c_{m}^{\prime} \\
b_{1} d_{1}^{\prime}, \ldots, b_{1} d^{\prime}{ }_{m}, \ldots, b, d_{1}^{\prime}, \ldots, b, d_{m}^{\prime}
\end{array}\right]=\left[\begin{array}{l}
a_{1} c_{1}, \ldots, a_{1} c_{n}, \ldots, a_{r} c_{1}, \ldots, a_{r} c_{n} \\
b_{1} d_{1}, \ldots, b_{1} d_{n}, \ldots, b_{q} d_{1}, \ldots, b_{,}, d_{n}
\end{array}\right] .
$$

Per dimostrare ciò, si osservi che $(90)$ sarà $:\left(c_{1}, \ldots, c_{n}\right)=\left(c_{1}{ }^{\prime}, \ldots, c_{m} \backslash S\right.$ e $\left(d_{1}^{\prime}, \ldots, d_{m}{ }^{\prime}\right)=\left(d_{1}, \ldots, d_{n}\right) S_{-1}$, ossia :

e perciò :

$$
\begin{aligned}
& c_{i}=\sum_{i}^{m}{ }_{j}^{m} c_{j}^{\prime} S_{j, i}^{\prime} \quad(i=1, \ldots, n) \\
& d_{i}{ }^{\prime}=\sum_{1}^{n} d_{j} S_{i, j} \quad(i=1, \ldots, m),
\end{aligned}
$$

$$
\begin{array}{rr}
a_{k} c_{i}=\sum_{1}^{m} a_{k} c_{j}{ }^{\prime} S_{i, j} & (i=1, \ldots, n) \\
b_{k} d_{i}=\sum_{1}^{n} b_{k} d_{j} S_{i, j} & (k=1, \ldots, r) \\
& (i=1, \ldots, m) \\
& (k=1, \ldots, r)
\end{array}
$$

pongasi :

$$
\begin{aligned}
a_{k} c_{i}^{\prime}=e_{(k-1) m+i}^{\prime} & (k=1, \ldots, r) \\
b_{k} d_{i}^{\prime}=f_{(k-1) n+1}^{\prime} & (i=1, \ldots, m) \\
a_{k} c_{i}=e_{(k-1) n+1} & (k=1, \ldots, r) \\
b_{k} d_{i}=f_{(k-1) n+1} & (i=1, \ldots, n)
\end{aligned}
$$


L'indice degli $e, f$ varia da 1 ad $n r$, quello degli $e^{\prime}, f^{\prime}$ da 1 ad $m r$; pongasi:

$$
\begin{array}{ll}
T_{(h-1) m+i,(k-1) n+j}=0 \text { se } h \neq k \\
T_{(k-1) m+i,(k-1) n+j}=S_{i, j}
\end{array} \quad\left(\begin{array}{l}
k=1, \ldots, r \\
h=1, \ldots, r \\
i=1, \ldots, m \\
j=1, \ldots, n
\end{array}\right) .
$$

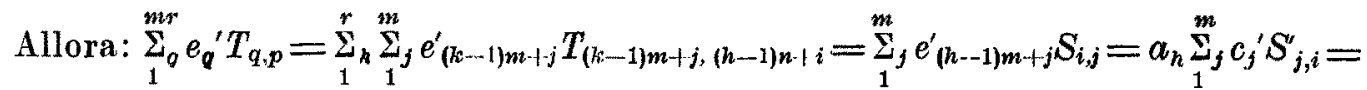

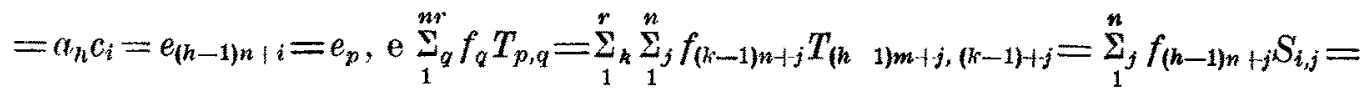
$=b_{n} \sum_{i}^{n} d_{j} S_{i, j}=b_{n} d_{i}^{\prime}=f^{\prime}(h-1) m-i=f_{p}^{\prime}$, e in forma matriciale, se

$$
T=\left(\begin{array}{ccccc}
T_{1,1} & \ldots & \ldots & T_{1 n, r} \\
\ldots & \ldots & \ldots & \ldots & \ldots \\
T_{m r, 1} & \ldots & \ldots & T_{m r, n r}
\end{array}\right),
$$

$\grave{e}$, con evidente significato dei simboli : $e=e^{\prime} T, f^{\prime}=f T_{-1}$, c. v. d. (90). Si vede allora immediatamente che le (37), (38), (39) sono soddisfatte, onde $J$, con le operazioni cosi definite, $̇ ̀$ un'algebra. Suppongasi ora che $A, B$ abbiano i moduli $u, v$ : allora le sub-algebre di $J$ formate dagli elementi $\left[\begin{array}{l}u \\ b\end{array}\right]^{\prime},\left[\begin{array}{l}a \\ v\end{array}\right]$ sono isomorfe a $B, A$.rispettivamente, e, se si indicano con $B^{\prime}, A^{\prime}$, si vede che $A^{\prime} B^{\prime}=J$, e che ogni elemento di $A$ commuta con ogni elemento di $B^{\prime}$. Poi, se $\left[\begin{array}{l}a_{1} \\ v\end{array}\right]\left[\begin{array}{l}u \\ b_{1}\end{array}\right]+\ldots+\left[\begin{array}{l}a_{v} \\ v\end{array}\right]\left[\begin{array}{l}u \\ b_{r}\end{array}\right]=0$, è $\left[\begin{array}{l}a_{1}, \ldots, a_{,} \\ b_{1}, \ldots, b_{r}\end{array}\right]=0$, e quindi, se non è $b_{1}=\ldots=$ $=b_{3}=0$, le $a_{1}, \ldots, a_{1}$. sono necessariamente fra loro dipendenti, per (90); quindi $J=A^{\prime} \times B^{\prime}$.

Siano poi $A, B$ due algebre qualsiasi, e siano $A_{1}, B_{1}$ due algebre con modalo contenenti le sub-algebre $A^{\prime}, \dot{B}^{\prime}$ isomorfe ad $A, B$ rispettivamente (86). Sia $J=A_{2} \times B_{2}$, con $A_{2}, B_{2}$ isomorfe rispettivamente ad $A_{1}, B_{1}$. Sia $D=A^{\prime \prime} B^{\prime \prime}$, con $A^{\prime \prime}, B^{\prime \prime}$ corrispondenti ad $A^{\prime}, B^{\prime}$, in $A_{2}, B_{2}$; è certo $D \neq 0$; inoltre, ogni elemento di $A^{\prime \prime}$ commuta con ogni elemento di $B^{\prime \prime}$; infine, se degli elementi di $A^{\prime \prime}$ sono dipendenti in $B^{\prime \prime}$, essi sono elementi di $A_{2}$ dipen. denti in $B_{2}$, onde sono dipendenti, e perciò $D=A^{\prime \prime} \times B^{\prime \prime}$. Così il (87) resta completamente dimostrato.

2.7. Proprietà del prodotto diretto. - Nell'ultima dimostrazione del $\mathrm{n}$. precedente è implicità anche la dimostrazione del

(92) Teorema. - Se $\mathrm{S} \times \mathrm{T}$ esiste, ed $\mathrm{S}^{\prime} \leq \mathrm{S}, \mathrm{T}^{\prime} \leq \mathrm{T}$, allora $\mathrm{S}^{\prime} \times \mathrm{T}^{\prime}$ esiste.

(93) Teorma. - Se esiste $\mathrm{S} \times(\mathrm{T}+\mathrm{U})$, esistono $\mathrm{S} \times \mathrm{T}$ ed $\mathrm{S} \times \mathrm{U}$, ed $\dot{e}$ $\mathrm{S} \times(\mathbf{T}+\mathrm{U})=\mathrm{S} \times \mathbf{T}+\mathbf{S} \times \mathbf{U}$. 
(94) Teorema. - Se $\mathrm{A}=\mathrm{B} \times \mathrm{C}$, valgono le seguenti proprietà :

a) Se $\mathrm{B}$ e $\mathrm{C}$ sono commutative, tale è $\mathrm{A}$.

b) Se $\mathrm{B}_{1}$ è una sub-algebra invariante sinistra (destro) in $\mathrm{B}$, e $\mathrm{C}_{1}$ in $\mathrm{C}$, tale $\grave{e} \mathrm{~B}_{1} \times \mathrm{C}_{1}$ in $\mathrm{A}$.

c) Se $\mathrm{t}$ è un intero maggiore di 0 , é $\mathrm{A}^{\mathrm{t}}=\mathrm{B}^{\mathrm{t}} \mathrm{C}^{\mathrm{t}}$.

d) A ̀̀ pseudonulla se, e solo se tale è almieno una delle $\mathrm{B}, \mathrm{C}$.

e) Se $\mathrm{B}$ è $\mathrm{C}$ sono dotate di indice, accade che:

se $\mathrm{B}$ e $\mathrm{C}$ non sono pseudonulle, l'indice di $\mathrm{A}$ è il maggiore fra quelli $d i \mathrm{~B} e \mathrm{C}$;

se $\mathrm{B}$ è pseudonulla, ma $\mathrm{C}$ non lo è, l'indice di $\mathrm{A}$ è uguale a quello di $A$; se $\mathrm{B}$ e $\mathrm{C}$ sono pseudonulle, lindice di $\mathrm{A}$ è $i l$ minore fra quelli $d i \mathrm{~B} e \mathrm{C}$.

f) Se A è semisemplice, tali sono $\mathrm{B} e \mathrm{C}$.

g) Se B e C hanno modulo, anche A lo ha, ed esso è il prodotto dei moduli di $\mathrm{B} e \mathrm{C}$.

h) $I l$ prodotto di un automodulo di $\mathrm{B}$, e di uno di $\mathrm{C}$,è un automodulo di $\mathrm{A}$.

\subsection{Proprietà degli automoduli.}

(95) Teonema. - Un'algebra dotata di un automodulo non e pseudonulla.

È evidente; non è però vero, in generale, come accade nelle algebre a base finita, il reciproco; anzi, esistono algebre prive di elementi pseudonulli e prive di nutomoduli. Si dirà algebra potenziale generata dall'elemento $x$ di $A$, l'algebra ogni cui elemento è combinazione degli elementi $x, x^{2}, x^{3} \ldots$. Se tali elementi sono fra loro indipendenti, l'algebra potenziale di $x$ non è di ordine finito, e viceversa.

(96) TEorema. - Se l'algebra potenziale dell'elemento x di A contiene il modulo u di A, essa è di ordine finito.

Se infatti $u=u_{1} x+\ldots+u_{1} x^{r}$, con $u_{1}, \ldots, u_{r}$ numeri, è anche $x=u_{1} x^{2}+$ $+\ldots+u, x^{r+1}$, e. v. d.

L'ordine dell'algebra potenziale di $x$ si dirà il grado di $x$.

(97) Teorema. - Se l'algebra A ha il modulo u e l'automodulo $\mathrm{v} \neq \mathrm{u}$, l'elemento $\mathrm{w}=\mathrm{n}-\mathrm{v}$ è un automodulo nullifico di $\mathrm{v}$; e ogni elemento che abbia in $\mathrm{v}(0 \mathrm{w})$ un modulo sinistro, o destro, ha in $\mathrm{w}$ (o v) un nullifico rispetliva. mente sinistro o destro.

(98) Teorema, - Se l'algebra A ha il modulo u. ogni antomodulo $\mathrm{v} \neq \mathrm{u}$ è un divisore destro e sinistro dello 0.

(99) Tuorema. - Se v è un automodulo di A, il sistema vA (rispelt. Av) è un'algebra, ed è l'insieme di tulti e soli ali elementi di A che hanno in $\mathrm{v}$ un modulo sinistro (rispett. destro).

(100) Tronema. - Se v è un aulomodulo di A, linsieme di tutti e soli gli elementi che hanno in $\mathrm{v}$ un modulo è $\mathrm{v} \mathrm{v}$.

(101) Teorema. - Se A ha il modulo $\mathrm{u}$ e l'automodulo $\mathrm{v} \neq \mathrm{u}$, e $\mathrm{w}=\mathrm{u}-\mathrm{v}$, l'insieme dei nullifici di $₹$ è l'algelore wAw. che ha il modulo w. 
(102) Teorema. - Automoduli di un'algebra, che siano a 2 a 2 nullifici, sono indipendenti, e la loro somma è un'automodulo che è un modulo di ciascuno di essi. (103) Teonema. - Se $\mathrm{u}, \mathrm{v}$ sono automoduli mutuamente nullifici, e se $\mathrm{u}=\mathrm{u}_{\mathbf{4}}+$ $+\ldots \mathrm{u}_{\mathrm{p}}$, con $\mathrm{u}_{1}, \ldots, \mathrm{u}_{\mathrm{p}}$ automoduli a 2 a 2 nullifici, ognuno di questi è nullifico di $\mathrm{v}$.

Siano ora $S, D, N$ i sistemi formati rispettivamente dai nullifici destri, sinistri, e dai nullifici dell'automodulo $v$; si vede subito che $v D \leq D$ è l'insieme degli elementi di $A$ ciascuno dei quali ha in $v$ un modalo sinistro ed un nullifico destro, e percio $v D \cap N=0$. Si ha che: $D=v D+N$, e analoga. mente $S=S v+N ; A=A v+D=v A+S$, onde, in definitiva: $A=A v+$ $+v D+N=v A v+S v+v D+N$, e ognuno dei sistemi $v A v, S v, v D, N$ è disginnto dalla somma degli altri; si ha percio il

(104) Teorema di Perrce. - Se $\mathrm{v}$ è un automodulo di A, ogni elemento di A si può porre, in un sol modo, sotto la forma $a=a_{1}+a_{2}+a_{3}+a_{4}$, ove $a_{1}$ ha in $\mathrm{v}$ un modulo, $\mathrm{a}_{2}$ un modulo sinistro e un nullifico destro, $\mathrm{a}_{3}$ un nullifico sinistro e un modulo destro, $\mathrm{a}_{4}$ un nullifico.

Si definiscono cosi, come per le algebre a base finita, il $1^{\circ}, 2^{\circ}, 3^{\circ}$ e $4^{\circ}$ sistema di Peirce, e si ha: $D A=A S=D S$. Si constata anche che $v A v$ ò un'algebra, e che $N$, se non è nullo, è un'algebra; $v D$ e $S v$, se non sono nulli, sono zero-algebre. L'automodulo $v$ si dirà primitivo se il $1^{\circ}$ sistema di PeIrce legato ad esso è privo di antomoduli diversi da $v$, e si dirà principale se il $4^{\circ}$ sistema di Peirce legato ad esso è privo di automoduli.

(105) Teorema. - Se u è un automodulo non primitivo di A, esiste in A almeno un automodulo $\mathrm{v} \neq \mathrm{u}$ tale che il $1^{\circ}$ sistema di Peirce legato a $\mathrm{v}$ sia una sub-algebra propria di quello legato ad $\mathrm{u}$.

Non è però necessariamente vero che un'algebra dotata di automoduli sia dotata di automoduli primitivi : sia infatti $A$ l'algebra in $O(2)$ ohe ha per base l'insieme degli elementi $x_{i}$ (i reale positivo), col prodotto così definito: $x_{i} x_{j}=x_{j} x_{i}=x_{j}$ se $j \geq i$; in essa ogni elemento è automodulo; considerato l'elemento $x_{y_{i}}+\ldots+x_{n}\left(r_{1}<\ldots<r_{n}\right)$, se $n$ è dispari, un antomodulo che abbia in esso un modulo è $x_{r_{n}+1}$, che è certo diverso dall' elemento dato, $e$ se $n$ è pari, ̀̀ $x_{n}+x_{r_{n}}$ ove $r_{n-1}<r<r_{n}$, che è pure diverso dall'eleménto dato.

$E$ non è neppure sempre vero che un'algebra dotata di automoduli sia dotata di automoduli principali; sia infatti $B$ l'algebra in $C(2)$ che ha per base $x_{1}, x_{2}, \ldots$, ove $x_{i}{ }^{2}=x_{i}$, e $x_{i} x_{j}=0$ se $i \neq j$; in questa ogni elemento è automodulo, ed ha certamente dei nullifici.

(106) Teorema. - L'unico automodulo principale di un'algebra con modulo è il modulo.

È evidente che non è sempre vero che un automodulo non primitivo sia sempre somma di automoduli primitivi a due a due nullifici; però :

(107) Teonema. - Un automodulo che sia somma di automoduli a due a due nullifici, non è primitivo. 
2.9. Algebre normali. - Se $G$ è un prolungamento del corpo $\Gamma$, esso si potrà considerare come un'algebra su $T$, ehe si potrà indicare ancora con $G$. Data un'algebra $A$ 'su $\mathrm{N}$, esiste (87) il prodotto diretto di due algebre isomorfe rispettivamente a $G, A$ su $\Gamma$; un'algebra $B$ sul corpo $G$ prolungamento di $\Gamma$, che contenga l'algebra $A$ su $\Gamma$ come sub-algebra, e che sia isomorfa so $\Gamma$ al prodotto diretto di due algebre $A^{\prime}, G^{\prime}$ isomorfe rispettivameute ad $A$ ed a $G$ considerato come algebra su $\Gamma$, in un isomorfismo in cui $A$ ed $A^{\prime}$ si corrispondono, si dirà una estensione di $A$ in $G$. Le considerazioni fatte assicu. rano che:

(108) Teorema. - Se G è un prolungamento del corpo $\Gamma$, e A è un'algebra su $\mathrm{\Gamma}$, e $\mathrm{B}$ una estensione di A in $\mathrm{G}$, se A ha una base, B ha la stessa base. (109) Teorema. - Se le algebre $\mathrm{A}, \mathrm{B}$ sono dotate di base, tale è $\mathrm{A} \times \mathrm{B}$, e se le basi sono ben ordinabili, tale è la base di $\mathrm{A} \times \mathrm{B}$.

Dim. - Se $T$ è la base di $A, S$ quella di $B$, l'insieme $V$ degli elementi di $A \times B$, ciascuno dei quali è prodotto di un elemento di $T$ e di uno di $S$, consta di elementi indipendenti, e forma una base di $A \times B$; il resto è palese, c. v. d.

Un'algebra si dirà normale se ha modulo, e se il suo centro coincide con l'algebra potenziale generata dal modulo.

(110) Teorema. - Se $\mathrm{A}=\mathrm{B} \times \mathrm{C}$, e $\mathrm{B}$, O hanno lo stesso modulo, il centro di $\mathrm{A}$ è il prodotto diretto del centro di $\mathrm{B}$ per quello di $\mathrm{C}$.

Dim. - Evidentemente, se $A_{1}, B_{1}, C_{1}$ sono i centri di $A, B, C$, ̀̀ $B_{1} \times C_{1} \leq A_{1}$. Sia poi $a$ un elemento di $A_{1}$; si può porre sotto la forma $a=c_{1} b_{1}+\ldots+c_{r} b_{r}$, con $\mathrm{i} c_{1}, \ldots, c_{0}$, indipendenti, ed $\mathrm{i} b, \ldots, b$, indipendenti ; per ogni elemento $b$ di $B$, dovrà essere $a b=b a$, ossia $c_{1} b b_{1}+\ldots+c, b b_{r}=c_{1} b_{1} b+\ldots+c_{r} b, b$, e cioè : $c_{1}\left(b b_{1}-b_{1} b\right)+\ldots+c_{r}\left(b b_{n}-b, b\right)=0$, onde $b_{1}, \ldots, b_{r}$. commutano con ogni elemento di $B$, e analogamente $c_{1}, \ldots, c_{2}$ con ogni elemento di $C$, e perciò $b_{1}, \ldots, b$, sono elementi di $B_{1}$, e $c_{1}, \ldots, c_{r}$ di $C_{1} ;$ quindi $A_{1} \leq B_{1} \times C$, e infine $A_{1}=B_{1} \times C_{1}$, c. v. a.

(111) Corollario. - Se $\mathrm{A}=\mathrm{B} \times \mathrm{C}$, con $\mathrm{B}$ nomale, e $\mathrm{B}, \mathrm{C}$ hanno lo stesso modulo, il centro di A coincide con quello di $\mathrm{C}$, e gli elementi di A che commutano con tutti quelli di $\mathrm{B}$, sono tutti e soli quelli di $\mathrm{C}$.

La seconda parte è implicita nella dimostrazione di (110).

(112) Corollario. - Il prodotto diretto di due algebre normali con lo stesso modulo è un'algebra normale, e gli elementi dell' uno sono tutti e soli gli elementi del prodotto che commutano con ogni elemento dell' allra.

(113) Teonema. - Se $\mathrm{A}=\mathrm{B} \times \mathrm{C}$ è normale, tali sono $\mathrm{B} e \mathrm{C}$.

2.10. Ulteriori proprietà dei ruri tipi di algebra. - Sia $A=A_{1}+\ldots+A_{2}$, e sia $v$ un automodulo di $A$; sarà $(79) v=v_{r_{1}}+\ldots+v_{r_{s}}$, con $s \leq r$, ove $v_{r}$, è un automodulo di $A_{r_{i}}$, nullifico di tutti i $v_{r_{j}}$ con $j \neq i$. Si ha allora che 
(114) Teorema. - Il $1^{\circ}$ sistema di Peirce legato a $\mathrm{v}$ è la somma diretta di quelli legati ai $\mathrm{v}_{\mathrm{r}_{\mathrm{i}}}$ nelle $\mathrm{A}_{\mathrm{r}_{\mathrm{j}}}$.

Poi :

(115). Teorema. - Il \{́ $^{\circ}$ sistema di Peirce legato a $\mathrm{v}$ in $\mathrm{A}$, è la somma diretta di quelli legati ai $\mathrm{v}_{\mathrm{v}_{\mathrm{i}}}$ nelle $\mathrm{A}_{\mathrm{r}_{\mathrm{i}}}$, e delle algebre $\mathrm{A}_{\mathrm{j}}$, ove $\mathrm{j}$ sia diverso $d a$ $\mathrm{r}_{1}, \ldots, \mathrm{r}_{\mathrm{s}}$.

Perciò :

(116) TeOREMA. - Se $\mathrm{A}=\mathrm{A}_{1}+\ldots+\mathrm{A}_{\mathrm{r}}$, gli automoduli primitivi di A sono tutti e soli quelli delle. $\mathrm{A}_{\mathrm{i}}$, e quelli principali di A sono tutti e soli quelli che sono somme di automoduli principali delle $\mathrm{A}_{\mathrm{i}}$.

(117) Twonwa. - Se $\mathrm{A}_{1}$ è una sub-algebra invariante propria di $\mathrm{A}$, ed $\mathrm{A}, \mathrm{A}_{1}$ sono dotale di modulo, A è somma diretta di $\mathrm{A}_{1}$, e di un'algebra $\mathrm{A}_{2}$, dotata di modulo e unica. Inoltre, $\mathrm{A}_{1} \dot{e}$ l'insieme degli elementi di $\mathrm{A}$ che hanno un modulo nel modulo di $\mathrm{A}_{1}$.

(118) Teorema. - Se A ha il modulo u, e u' $\mathrm{u}^{\prime}$ u è un automodulo per cui sia $\mathrm{n}^{\prime} \mathrm{A}=\mathrm{Au}^{\prime}, \mathrm{A}$ è riducibile.

(119) Criterio di Scmeffers. - Condizione necessaria e sufficiente affinchè uu'algebra con modulo sia riducibile, è che possieda un automodulo diverso dal modulo, che commuti con ogni elemento dell'algebra.

(120) TEOREMA. - Se A è sommatoria ed ha modulo, e $\mathrm{A}=\mathrm{B}+\mathrm{C}$, con $\mathrm{B}, \mathrm{C}$ sistemi non nulli, tali che $\mathrm{BC}=\mathrm{CB}=\mathrm{C}, \mathrm{A}$ è riducibile, e $\mathrm{B}, \mathrm{C}$ sono sub-al. gebre invarianti proprie di A.

(121) Teorema. - Un'algebra riducibile, sommatoria, e dotata di modulo, se è decomponibile nella somma diretta di un numero finito di algebre irriducibili, lo è in un sol modo.

(122) T'eовема. - Se un'algebra commutativa con modulo è decomponibile nella somma diretta di algebre irriducibili, il numero di tali algebre è uguale al numero degli automoduli primitivi dell'algebra data.

Non è però detto che un'algebra commutativa con modulo abbia un nu. mero finito di automoduli primitivi, o ohe, essendo ciò verificato, sia somma dirutta di algebre irriducibili (cfr. algebre booleane).

(123) Teorema. - Se un'algebra è primitiva e dotata di modulo, e l' equazione minima di un suo elemento dotato di equazione minima è $\mathbf{f}(\mathbf{x})=0, \mathbf{f}(\mathbf{x})$ è un polinomio irriducibile nel corpo in cui l'algebra è definita.

(124) 'Teorema. - Se un'algebra con modulo è irriducibile e non semplice, ogni sua sub-algebra invariante propria è priva di modulo.

Non è però vero, come nelle algebre a base finita, che un'algebra semisemplice, ma non semplice, sia riducibile.

(125) Teorema. - Un'algebra A semisemplice, ma non semplice, che sia somma diretta delle algebre semplici $\Lambda_{1}, \ldots, A_{t}$, non ha altre sub-algebre invarianti proprie all' infuori delle $2^{\mathrm{t}}-2$ sub-algebre date $d a \mathrm{~A}_{1}, \ldots, \mathrm{A}_{\mathrm{t}}$ e $\left(\begin{array}{ll}\mathrm{g} & \mathrm{t}>2\end{array}\right)$ delle somme dirette di un numero qualsiasi di esse. 
(126) Teorema. - Un'algebra semplice e commutativa ha modulo, purchè non sia una zero-algebra.

Dim. - Se $A$ non avesse modulo, ogni suo elemento dorrebbe essere (60), (45) singolare sinistro e destro, o divisore sinistro e destro dello 0 . Ed allora, se $a$ è un elemento singolare di $A$, ̀े $a A=A a<A$; ma $a A$ è invariante in $A$, onde $a A=0$; se invece $a$ non è singolare, l'algebra $B$ dei suoi nullifici esiste, e, poichè è invariante in $A$, sarà $B=A$, onde in ogni caso $a A=A a=0$, contro l'ipotesi che $A$ non sia una zero algebra, c.. . d.

(127) Teorema. - Un'algebra semplice, primitiva, e priva di modulo, è priva di centro.

Dim. - Se infatti $a \neq 0$ fosse un elemento del centro, ripetendo il ragionamento precedente si vedrebbe che a dovrebbe essere singolare, e perciò $a A=A a=0$, onde $A$ non sarebbe primitiva, c. v. d.

(128) Teorema. - Il $1^{\circ}$ sistema di Peirce legato ad un automodulo di un'algebra semplice con modulo è un' algebra semplice.

Dim. - Sia $v$ l'automodulo, e $B$ una sub-algebra invariante di $v A v$; è $(v A v) B(v A v)=v(A B A) v \leq B ;$ ma $A B A \geq B>0$, onde $A B A=A$, e perciò $v A v \leq B \leq v A v$, c. v. d. 